\title{
The luminous blue variable HR Carinae has a partner ${ }^{\star}$ Discovery of a companion with the VLTI
}

\author{
Henri M. J. Boffin ${ }^{1,2}$, Thomas Rivinius ${ }^{1}$, Antoine Mérand ${ }^{1}$, Andrea Mehner ${ }^{1}$, Jean-Baptiste LeBouquin ${ }^{3}$, \\ Dimitri Pourbaix ${ }^{4, \star \star}$, Willem-Jan de Wit ${ }^{1}$, Christophe Martayan ${ }^{1}$, and Sylvain Guieu ${ }^{1,3}$ \\ ${ }^{1}$ ESO, Alonso de Córdova 3107, Casilla 19001, Santiago, Chile \\ e-mail: hboffin@eso.org \\ 2 ESO, Karl-Schwarzschild-str. 2, 85748 Garching, Germany \\ ${ }^{3}$ Institut de Planétologie et d'Astrophysique de Grenoble (UMR 5274), BP 53, 38041 Grenoble Cedex 9, France \\ ${ }^{4}$ Institut d'Astronomie et d'Astrophysique, Université Libre de Bruxelles (ULB), 1050 Bruxelles, Belgium \\ Received 16 June 2016 / Accepted 18 July 2016
}

\begin{abstract}
Luminous blue variables (LBVs) are massive stars caught in a post-main sequence phase, during which they lose a significant amount of mass. Since, on one hand, it is thought that the majority of massive stars are close binaries that will interact during their lifetime, and on the other, the most dramatic example of an LBV, $\eta$ Car, is a binary, it would be useful to find other binary LBVs. We present here interferometric observations of the LBV HR Car done with the AMBER and PIONIER instruments attached to ESO's Very Large Telescope Interferometer (VLTI). Our observations, spanning two years, clearly reveal that HR Car is a binary star. It is not yet possible to fully constrain the orbit, and the orbital period may lie between a few years and several hundred years. We derive a radius for the primary in the system and possibly also resolve the companion. The luminosity ratio in the $H$-band between the two components is changing with time, going from about 6 to 9 . We also tentatively detect the presence of some background flux which remained at the $2 \%$ level until January 2016, but then increased to 6\% in April 2016. Our AMBER results show that the emission line-forming region of $\mathrm{Br} \gamma$ is more extended than the continuum-emitting region as seen by PIONIER and may indicate some wind-wind interaction. Most importantly, we constrain the total masses of both components, with the most likely range being $33.6 M_{\odot}$ and $45 M_{\odot}$. Our results show that the LBV HR Car is possibly an $\eta$ Car analog binary system with smaller masses, with variable components, and further monitoring of this object is definitively called for.
\end{abstract}

Key words. instrumentation: interferometers - binaries: visual - stars: individual: HR Car - stars: massive stars: variables: S Doradus

\section{Introduction}

Luminous blue variables (LBVs) are post-main-sequence massive stars undergoing a brief, but essential, phase in their life, characterised by extreme mass loss and strong photometric and spectroscopic variability (Conti 1984; Humphreys \& Davidson 1994; Vink 2012). The best known LBV, $\eta$ Carinae, is known to have lost about 10-30 solar masses during its great outburst in the 1840s (Smith et al. 2003). There is, as yet, no firmly established mechanism to explain the large mass loss of LBVs, nor how it happens: is the mass lost due to a steady radiatively-driven stellar wind, or is it removed by punctuated eruption-driven mass loss, such as the great outburst of $\eta$ Car? Numerous hypotheses have been proposed. Apart from single star processes, such as core and atmospheric instabilities (Humphreys \& Davidson 1994) or supercritical rotation, the binary hypothesis (e.g. Gallagher 1989; Smith 2011) is a very strong contender, especially since it is well established that massive stars form nearly exclusively in multiple systems and that binary interactions are critical for these stars (Chini et al. 2012; Sana et al. 2012, 2014).

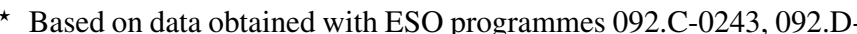
0289, 092.D-0296, 094.D-0069, and 596.D-0335.

$\star \star$ Senior Research Associate, F.R.S.-FNRS, Belgium.
}

There is currently a hot debate in the literature on the evolutionary status of LBV stars and on the importance of binarity in their formation (Smith \& Tombleson 2015; Humphreys et al. 2016). So far, however, while several wide LBV binaries were identified, LBV systems similar to $\eta$ Car (relatively close and eccentric) have not been found (Martayan et al. 2012, 2016). The only possible exception might be the LBV candidate MWC 314 (Lobel et al. 2013), but this is apparently a massive semidetached binary system, and thus not directly comparable to $\eta$ Car. On the one hand, this may appear rather surprising since it is thought that given their very high multiplicity rate, more than $70 \%$ of all massive stars exchange mass with a companion (Sana et al. 2012). On the other hand, LBVs are rare objects with complex emission line spectra and intricate nebulae. Located at average distances of a few kpc or more, they therefore require at least milli-arcsecond resolution for direct close companion detection. This level of resolution is only reachable by interferometry.

Here, we report on interferometric measurements of the LBV HR Carinae (HD 90177), one of the very few in the Milky Way. Van Genderen et al. (1990) derived for this star an effective temperature of $14000 \pm 2000 \mathrm{~K}$ and a bolometric luminosity $\left(M_{\mathrm{bol}}\right)$ of -9.5 , with a mass-loss rate of $2.2 \times 10^{-5} M_{\odot} \mathrm{yr}^{-1}$. The luminosity was revised to $M_{\mathrm{bol}}=-8.9$ and the distance to $5 \pm 1 \mathrm{kpc}$ 
by van Genderen et al. (1991), putting HR Car most likely in the Carina spiral arm. At the same time, Hutsemékers \& van Drom (1991) derived a kinematic distance of $5.4 \pm 0.4 \mathrm{kpc}$ and showed that HR Car has a multiple shell expanding atmosphere. Weis et al. (1997) found that HR Car has a nebula that appears bipolar, with each lobe having a diameter of $\sim 0.65 \mathrm{pc}$ and a lineof-sight expansion velocity of $75-150 \mathrm{~km} \mathrm{~s}^{-1}$. We note in passing that the HIPPARCOS measurement of the parallax of HR Car of $1.69 \pm 0.82$ mas (van Leeuwen 2007), translating to a very imprecise distance of $592_{-193}^{+557} \mathrm{pc}$, is most likely incorrect in view of the other indicators, and quite possibly a result of the hitherto unknown binarity.

Effective temperature determinations for HR Car range between about $10000 \mathrm{~K}$ (Machado et al. 2002), $14000 \pm 2000 \mathrm{~K}$ (see above), $17900 \mathrm{~K}$ (Groh et al. 2009), and $22000 \mathrm{~K}$ (Hohle et al. 2010). The star is highly variable: it had its last S Dor outburst in July 2001 and is currently in a quiet state, two magnitudes fainter than at maximum (in $V$ ). Visual magnitudes obtained on the AAVSO website indicate that the star now has a magnitude $V \sim 8.7-9$. According to Tetzlaff et al. (2011), HR Car is a B2evar star with a mass of $18.1 \pm 5.5 M_{\odot}$ and an age of $5.0 \pm 1.4 \mathrm{Myr}$, while Hohle et al. (2010) quote a value of $23.66 \pm 7.24 M_{\odot}$. Similarly, Groh et al. (2009) show the star to have a high rotational velocity of $150 \pm 20 \mathrm{kms}^{-1}$, i.e. rotating at $88 \%$ of its critical velocity, and derive a current mass of about $25 M_{\odot}$, and an initial mass of $50 \pm 10 M_{\odot}$, but we should stress here that all these values are very model-dependent. The high velocity and the difficulty of LBVs to lose angular momentum led Groh et al. (2009) to suggest that HR Car could explode during its current LBV phase, making the link with detections of LBVlike progenitors of Type IIn supernovae (Smith \& Tombleson 2015). It is thus important to characterise, as well as possible, this very interesting star.

We show here, based on interferometric measurements, that the LBV HR Car is in fact a binary system with an orbital period of several years, therefore making it the first LBV similar to $\eta$ Car in terms of binarity. Our observations are presented in Sect. 2 and discussed in Sect. 3.

\section{Observations}

\section{1. $A M B E R$}

The LBV HR Car was observed as part of the OHANA survey (Rivinius et al. 2016), which secured spectrally resolved 3-beam interferometry of Br $\gamma$ with AMBER (Petrov et al. 2007) at ESO's Very Large Telescope Interferometer (VLTI). In addition, a single previous AMBER observation of HR Car was obtained from the archive. All AMBER observations were reduced the standard way, i.e. with amdlib ${ }^{1}$, in version 3.0.6 (Chelli et al. 2009). A summary of the observations is given in Table 1 . The way the OHANA survey was designed, no dedicated calibrators were taken and hence the visibility and phase observations are normalised to the local continuum. Owing to the backup and snapshot nature of the OHANA survey, the data quality is not very homogeneous, as can be seen in Fig. 1, where some of the AMBER observations are shown.

The observed $\operatorname{Br} \gamma$ line profile is typical for strong LBV winds, with a P Cyg-type absorption seen at about zero and negative velocities, although not descending below the level of the continuum, and the peak of the emission somewhat redshifted.

The visibility signature, in first order a measure of the size of the emission region, is well centred on the overall emission.

\footnotetext{
1 http://WWW . jmmc . fr/data_processing_amber
}

Table 1. Log of AMBER observations.

\begin{tabular}{lll}
\hline \hline Date & Stations & MJD \\
\hline April 25, 2010 & U1-U2-U4 & 55311.024 \\
January 10, 2014 & A1-B2-C1 & 56667.361 \\
January 19, 2014 & A1-C1-D0 & 56676.226 \\
February 8, 2014 & D0-H0-G1 & 56696.343 \\
February 9, 2014 & A1-B2-C1 & 56697.284 \\
February 12, 2014 & A1-B2-C1 & 56700.172 \\
February 22, 2014 & D0-H0-I1 & 56710.103 \\
March 2, 2014 & D0-H0-I1 & 56718.085 \\
March 2, 2014 & G1-I1-H0 & 56718.149 \\
March 10, 2014 & A1-G1-K0 & 56726.113 \\
\hline
\end{tabular}

At the shortest baselines, shown in panel c, the visibility drop is too small to be seen, but along the NE-SW axis it becomes noticeable at about 30 to $40 \mathrm{~m}$ baseline length, and the visibility reaches zero at above $100 \mathrm{~m}$ baseline length. In the $K$-band, this means the line emission region of $\mathrm{Br} \gamma$ is of the order of 5 mas in size in this direction. Only a few measurements were aligned in the NW-SE direction, but in panel d of Fig. 1, the drop in visibility is clearly stronger than on similar baseline lengths in the perpendicular direction, as shown in panels a and $\mathrm{c}$. This means that the $\operatorname{Br} \gamma$ wind emission is not spherical in size, and more extended along the NW-SE axis.

In first order approximation, wavelength-differential phase, the second interferometric observable, is a measure of photocentre shift. A differential phase signature in the emission line is observed along the NE-SW axis, but not along the NW-SE axis, meaning that in the NW-SE direction the Br $\gamma$ emission is symmetric to the center-of-light in the continuum. In the NE-SW direction, however, it is offset towards the NE. Other than the visibility profile, the phase signature is also not centered on the emission in wavelength, but shifted to the blue. In other words, the part of the emission that is offset to the NE is also approaching the observer.

The signatures of phase and visbility observed in the OHANA survey are temporally stable, i.e. over the several months of observations. In particular the phase signature, which hallmarks a photocenter displacement, is hard to explain with the variable, yet more-or-less symmetric wind of a single supergiant star.

On longer timescales, however, there are changes. One of the three baselines observed in 2010 was at a similar $u, v$ position as some of those observed in 2014, see Fig. 1 panels a and e vs. c. Even though the quality of the data was lower in 2010, there was a clear visibility drop at this $u, v$ position, but little-to-no phase signature in 2010 while, in 2014, this was quite the opposite.

The above description is hard to reconcile with a single star model, and prompted further observations with another interferometric instrument, PIONIER, to test a binary hypothesis (see Rivinius et al. 2015, for a discussion of the alternatives and initial justification of the binary hypothesis).

\subsection{PIONIER}

We observed HR Car with the four 1.8-m Auxiliary Telescopes of the VLTI, using the PIONIER visitor instrument (Berger et al. 2010; Le Bouquin et al. 2011) in the $H$-band on the nights of 1-2, and 2-3 March 2014. This provided us with strong hints of the binarity of HR Car, and we therefore started to observe it on a regular basis. The log of our observations is shown in Table 2. In 2014, we used the prism in low resolution (SMALL), 


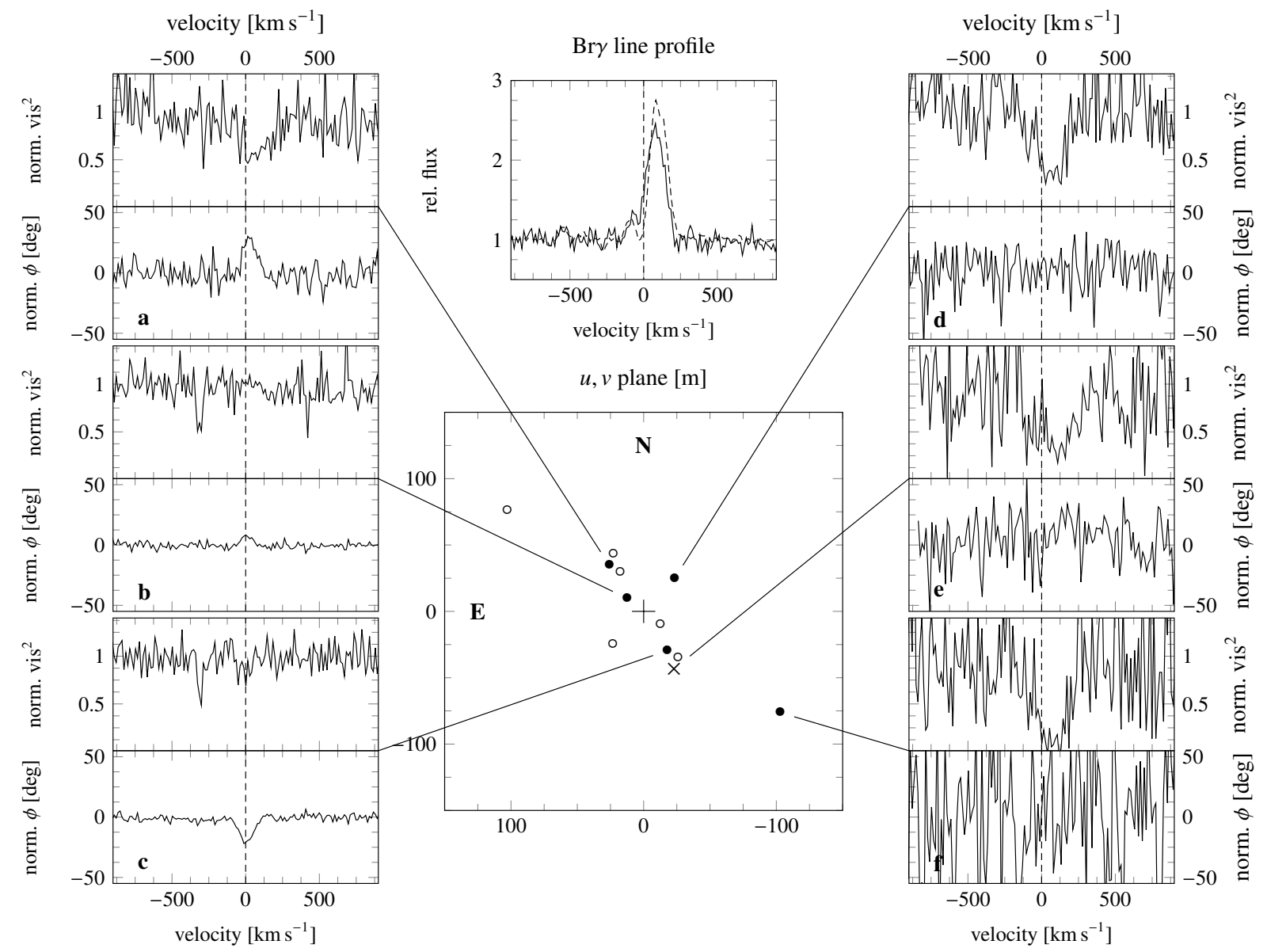

Fig. 1. Exemplary AMBER observations of HR Car. In the $u, v$-plane coverage, shown in the centre, solid lines connect visibility and phase observation with their respective $u, v$ points, open circles are conjugated $u, v$ points. Panels a) and d) show observations obtained on MJD 56 718.149, panels b) and c) on MJD 56 676.226, and panel f) on MJD 56 726.113. One baseline from the observation taken on MJD 55311.024 is shown as a dashed line in the flux panel and in panel $\mathbf{e})$, its $u, v$ position is indicated by a cross.

Table 2. Log of the PIONIER observations.

\begin{tabular}{lccl}
\hline \hline Date & $V^{2}$ points & CP points & MJD \\
\hline March 2, 2014 & 53 & 35 & 56718.254 \\
March 3, 2014 & 18 & 12 & 56719.047 \\
January 26, 2015 & 252 & 168 & 57048.310 \\
January 5, 2016 & 102 & 68 & 57392.337 \\
February 1, 2016 & 90 & 60 & 57419.118 \\
April 3, 2016 & 71 & 48 & 57482.060 \\
\hline
\end{tabular}

Notes. We indicate the time of observation, the number of visibilities and closure phases obtained, as well as the mean value of the epoch of observation.

the fringes being sampled over three spectral channels and the intermediate VLTI configuration D0-H0-I1-G1 was used. In 2015, we used the extended configuration A1-G1-K0-I1 and in 2016 the A0-G1-J2-J3 one. Early 2015, the detector of PIONIER was upgraded and, from then on, we used the GRISM mode, where the fringes are sampled over six spectral channels. The stars HD 90074, HD 87238, and HD 90980 were used as calibrators, in successive CAL-SCI-CAL sequences, with five SCI images taken during each sequence. Since we are using four telescopes, generally we have six visibilities and four closure phases (times the spectral channels) for each observation. Since we only had a few data points on the night of 2-3 March 2014, we have combined them with those obtained the night before.
Data reduction was done in the usual way with the pndrs ${ }^{2}$ package presented by Le Bouquin et al. (2011). We show the resulting squared visibilities and closure phases for the best data set - the one from January 2015 - in Fig. 2.

\section{Analysis and discussion}

The visibilities and closure phases obtained at the various epochs clearly reveal asymmetries that cannot be due to a single, spherical object, as shown by Fig. 2. We used the LITpro software ${ }^{3}$ (Tallon-Bosc et al. 2008) to model the PIONIER data. Using the data of January 2015 (i.e. those with the most points), we tested several models, and it was clear that we needed to have at least two components to best fit the data - this is obvious from the clear signal in the closure phases. In Table 3, we show the results for the best models we found: two point-like sources, two discs, the same when adding a background source, as well as a case for a point-like source, a disc and a background. It is clear that going from two points to two discs largely reduces the $\chi^{2}$ (from 3.6 to 1.8), while adding some background flux still decreases it to 1.5. Thus, for the continuum data observed by PIONIER, the best fit is given by two discs with a tiny background component. Figure 3 shows the reduced $\chi^{2}$ maps for the diameters of

\footnotetext{
2 http://www.jmmc.fr/data_processing_pionier.htm

3 LITpro is available from http://www.jmmc.fr/litpro_page.
} htm 
Table 3. Parameters of the best LITpro models to the PIONIER data.

\begin{tabular}{|c|c|c|c|c|c|c|c|c|}
\hline \multirow[t]{2}{*}{ Date } & \multirow[t]{2}{*}{ Model type } & \multirow{2}{*}{$\begin{array}{l}\text { Primary } \\
\text { diameter }\end{array}$} & \multirow[b]{2}{*}{ flux fraction } & \multicolumn{2}{|c|}{ Secondary } & \multirow[b]{2}{*}{$y$} & \multirow{2}{*}{$\begin{array}{l}\text { Background } \\
\text { flux fraction }\end{array}$} & \multirow[t]{2}{*}{$\chi_{r}^{2}$} \\
\hline & & & & diameter & $x$ & & & \\
\hline Mar. 2014 & 2 discs + bcg & $0.37 \pm 0.20$ & $14 \pm 1.5 \%$ & $1.03 \pm 0.41$ & $-1.08 \pm 0.03$ & $-1.70 \pm 0.03$ & $1.2 \pm 0.4 \%$ & 1.0 \\
\hline \multirow[t]{5}{*}{ Jan. 2015} & 2 points & - & $14.0 \pm 0.6 \%$ & - & $-1.54 \pm 0.01$ & $-0.71 \pm 0.01$ & - & 3.6 \\
\hline & 2 discs & $0.59 \pm 0.03$ & $13.8 \pm 0.7 \%$ & $1.23 \pm 0.09$ & $-1.55 \pm 0.01$ & $-0.76 \pm 0.01$ & - & 1.8 \\
\hline & 2 points + bcg & - & $12.6 \pm 0.6 \%$ & - & $-1.52 \pm 0.01$ & $-0.70 \pm 0.01$ & $3.2 \pm 0.2 \%$ & 1.6 \\
\hline & 1 point $+\operatorname{disc}+b c g$ & - & $14 \pm 0.6 \%$ & $1.07 \pm 0.09$ & $-1.50 \pm 0.01$ & $-0.69 \pm 0.01$ & $2.5 \pm 0.2 \%$ & 1.55 \\
\hline & 2 discs + bcg & $0.39 \pm 0.04$ & $12.9 \pm 0.6 \%$ & $0.76 \pm 0.15$ & $-1.55 \pm 0.01$ & $-0.73 \pm 0.01$ & $2.2 \pm 0.2 \%$ & 1.5 \\
\hline Jan. 2016 & 2 discs + bcg & $0.45 \pm 0.09$ & $13 \pm 3 \%$ & $0.86 \pm 0.22$ & $-0.68 \pm 0.07$ & $0.89 \pm 0.09$ & $1.6 \pm 0.4 \%$ & 0.8 \\
\hline Feb. 2016 & 2 discs $+b c g$ & $0.30 \pm 0.09$ & $16 \pm 2 \%$ & $0.86 \pm 0.22$ & $-0.53 \pm 0.02$ & $0.91 \pm 0.03$ & $3.5 \pm 0.4 \%$ & 0.8 \\
\hline Apr. 2016 & 2 discs + bcg & $0.37 \pm 0.04$ & $9.0 \pm 0.6 \%$ & $0.87 \pm 0.11$ & $-0.39 \pm 0.01$ & $1.35 \pm 0.03$ & $4.7 \pm 0.3 \%$ & 0.6 \\
\hline
\end{tabular}

Notes. The table gives the angular diameter of the primary, and for the secondary, its relative flux fraction, angular diameter, and its position, as well as the background flux, if present. All dimensions are given in mas.

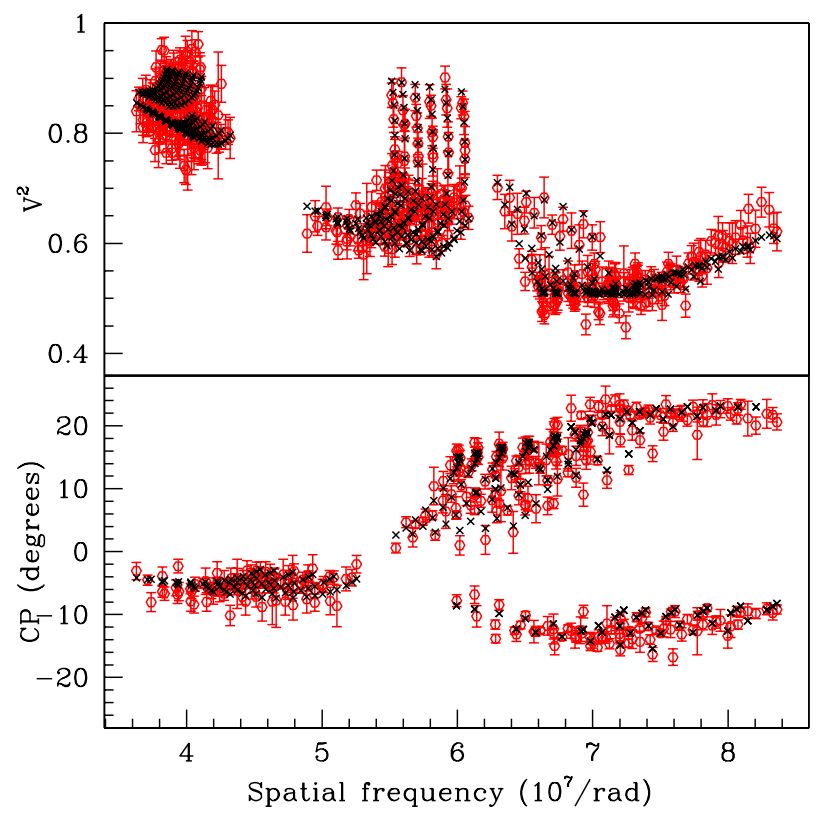

Fig. 2. PIONIER observations of HR Car in January 2015: squared visibilities (top) and closure phases (bottom). The data are shown as red crosses with error bars, while the best-fit model of two discs on top of a background is shown with black crosses. A single star would have bijective visibilities and a $0 \pm 180$ deg closure phase.

the two components, as well as for the flux of the background. This shows the confidence intervals of these parameters. From these as well as from Table 3, it is clear that, while the fluxes and the diameter of the primary are rather well constrained, that of the secondary is not, with errors of $20 \%$ for the January 2015 dataset. We come back to this below. We note that the relative positions of the two components is independent (within $3 \sigma$ ) of the model considered. This makes the derivation of the orbit (see below) very robust.

We then applied the same model to all our epochs to measure the diameter of the two discs and the relative fluxes and positions (see Table 3). For the best model, the diameters of the two components are relatively stable, which leads us to think that they are possibly physical. The relative flux fraction of both the secondary and the background does appear to change with time and we discuss this later.

We also used the CANDID $\operatorname{code}^{4}$ (Gallenne et al. 2015), a set of Python tools that was specifically created to search systematically for high-contrast companions. Although LITpro and CANDID are both based on a Levenberg-Marquardt minimisation, CANDID performs a systematic exploration of the parameters while LITpro only does one fit, and CANDID takes into account bandwidth smearing ${ }^{5}$. CANDID provided the best fit when using a disc for the primary, an unresolved secondary, and some background flux. The results for the various epochs are given in Table $4-$ it is clear that the derived values are in agreement with those found by LITpro, typically within $1 \sigma$. For the March 2014 epoch, the data were not good enough for CANDID to converge on a primary diameter and we fixed its value to the mean of the other epochs.

From this analysis, we are thus confident that the PIONIER data indicate:

- that there are two objects in the system;

- that they are moving relative to each other in an apparent orbital motion;

- that the relative flux fraction of the secondary in the $H$-band is about $15 \pm 1 \%$ from March 2014 till February 2016, and thus that the flux ratio between the two components is $5.6 \pm$ 0.5 , but that it dropped to $10 \%$ in April 2016 (flux ration of 9);

- that the primary has a diameter (in the $H$-band continuum) of $0.38 \pm 0.07$ mas;

- that there is also some background flux, amounting to 2-3\% until January 2016, but increasing since February 2016 to reach about $5-6 \%$.

\footnotetext{
4 https://github.com/amerand/CANDID

5 But this latter aspect should not make much difference in the case of HR Car.
} 
Table 4. Parameters of the best CANDID models to the PIONIER data.

\begin{tabular}{lcccccc}
\hline \hline Date & $\begin{array}{c}\text { Primary } \\
\text { diameter }\end{array}$ & $\begin{array}{c}\text { Background } \\
\text { flux fraction }\end{array}$ & flux fraction & $x$ & $y$ & $\chi_{r}^{2}$ \\
\hline Mar 2014 & $0.38^{a}$ & $1.7_{-0.9}^{+1.2} \%$ & $16.4_{-0.8}^{+1.0} \%$ & $-1.06_{-0.04}^{+0.04}$ & $-1.70_{-0.07}^{+0.07}$ & 2.30 \\
Jan 2015 & $0.45_{-0.05}^{+0.07}$ & $2.7_{-0.6}^{+0.6} \%$ & $14.3_{-0.4}^{+0.3} \%$ & $-1.57_{-0.01}^{+0.01}$ & $-0.74_{-0.02}^{+0.03}$ & 1.53 \\
Jan 2016 & $0.34_{-0.12}^{+0.17}$ & $2.2_{-0.6}^{+0.7} \%$ & $12.2_{-5.7}^{+3.8} \%$ & $-0.79_{-0.11}^{+0.14}$ & $0.93_{-0.22}^{+0.13}$ & 1.45 \\
Feb 2016 & $0.37_{-0.11}^{+0.13}$ & $4.3_{-0.6}^{+0.7} \%$ & $14.1_{-1.9}^{+1.3} \%$ & $-0.58_{-0.05}^{+0.05}$ & $1.02_{-0.04}^{+0.05}$ & 1.30 \\
Apr 2016 & $0.37_{-0.05}^{+0.07}$ & $5.8_{-0.5}^{+0.6} \%$ & $9.9_{-0.5}^{+0.4} \%$ & $-0.41_{-0.02}^{+0.02}$ & $1.35_{-0.04}^{+0.04}$ & 0.65 \\
\hline
\end{tabular}

Notes. The table gives the angular diameter of the primary, the background flux, and for secondary, its relative flux fraction and its position. The last column is the reduced $\chi^{2}$ of the model. All dimensions are given in mas. ${ }^{(a)}$ Parameter fixed.
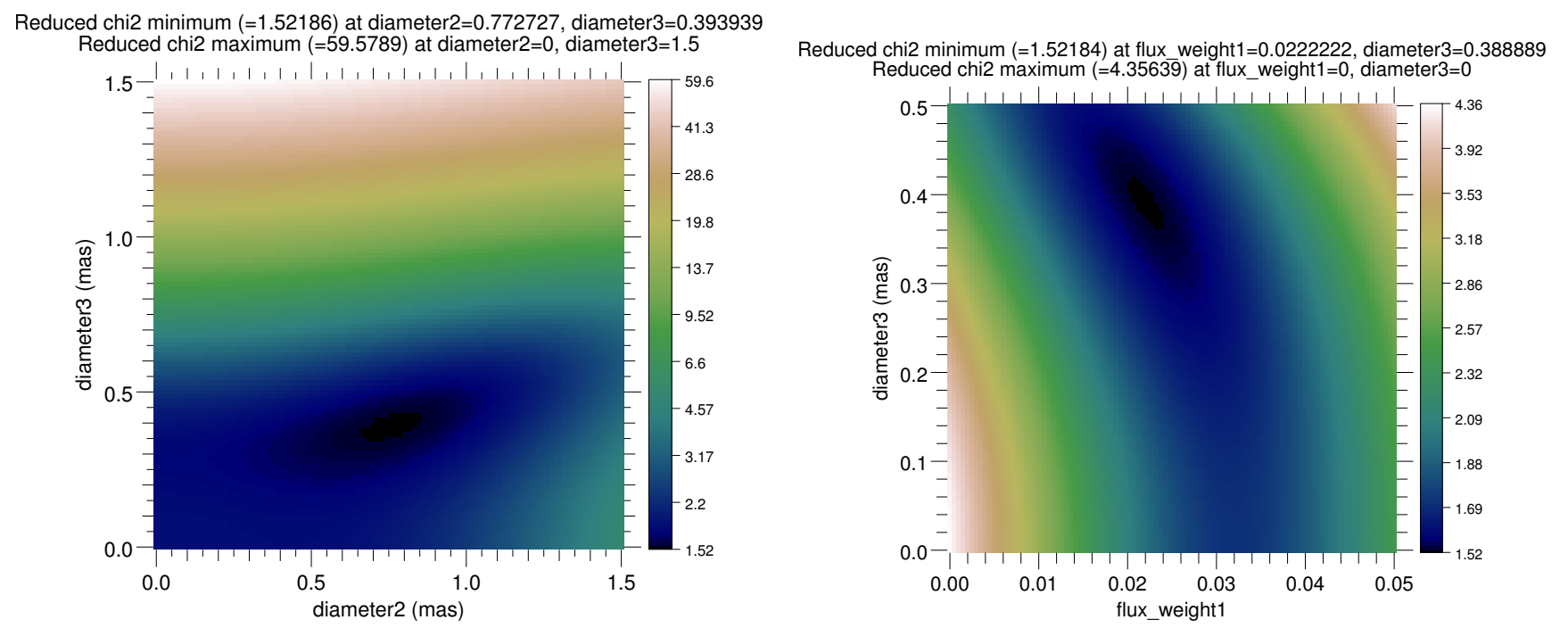

Fig. 3. Right: reduced $\chi^{2}$-map in the diameter-diameter plane for the two-disc+background LITpro model for the January 2015 data. Left: same, but in the primary disc diameter-background flux plane. The maps show the confidence interval for the best-fit parameters.

In addition, it is possible that the secondary is also resolved, although the disagreement between LITpro and CANDID on this indicates that the utmost caution is required when considering this possibility. If it is resolved, it has a diameter of $0.85 \pm 0.20$ mas, i.e. the less luminous component is more than twice as big as its companion. We will now discuss in turn what we can derive from these facts.

\subsection{Orbit}

Although our derived positions that change with time clearly reveal that HR Car is a binary, we do not yet have enough data to fully constrain the orbit. Our data points, as obtained with CANDID, are shown in Fig. 4, together with the best orbit that fits our PIONIER data points, i.e. the one with the smallest $\chi^{2}$. The corresponding parameters are given in Table 5. This orbit has an orbital period of $12 \mathrm{yr}$ and an eccentricity of 0.4 . At a distance of $5.4 \mathrm{kpc}$, the semi-major axis of this solution is $18 \mathrm{au}$.

However, this is only one possible orbit, as clearly shown by Fig. 5, which shows the result from a grid-search for all possible orbits, looking for the minimal $\chi^{2}$. From this, as well as from
Table 5. Parameters of the best orbit that fits the PIONIER points.

\begin{tabular}{lc}
\hline \hline$\Omega($ deg) & $46.9 \pm 0.6$ \\
Orbital period (days) & $4557.5 \pm 21.0$ \\
$T_{0}(\mathrm{MJD})$ & $56990.6 \pm 16.0$ \\
semi-major axis (mas) & $3.324 \pm 0.026$ \\
eccentricity & $0.4 \pm 0.2$ \\
inclination (deg) & $119.2 \pm 0.7$ \\
$\omega($ deg) & $201.9 \pm 2.1$ \\
\hline
\end{tabular}

Fig. 6, it appears that for now we cannot constrain the period and solutions between a few years up to several hundred years are possible. Of course, all parameters are not free and it is obvious that the long period orbits need to be very eccentric, a condition that is not required for shorter periods. In fact, given the arc we already have, we can be confident that the (deprojected) periastron is quite well defined around 2 mas, as is obvious from the second panels of Fig. 6 - except for those with very short semi-major axis and high masses, but these are also those less likely in terms of $\chi^{2}$. This corresponds at a nominal distance of 


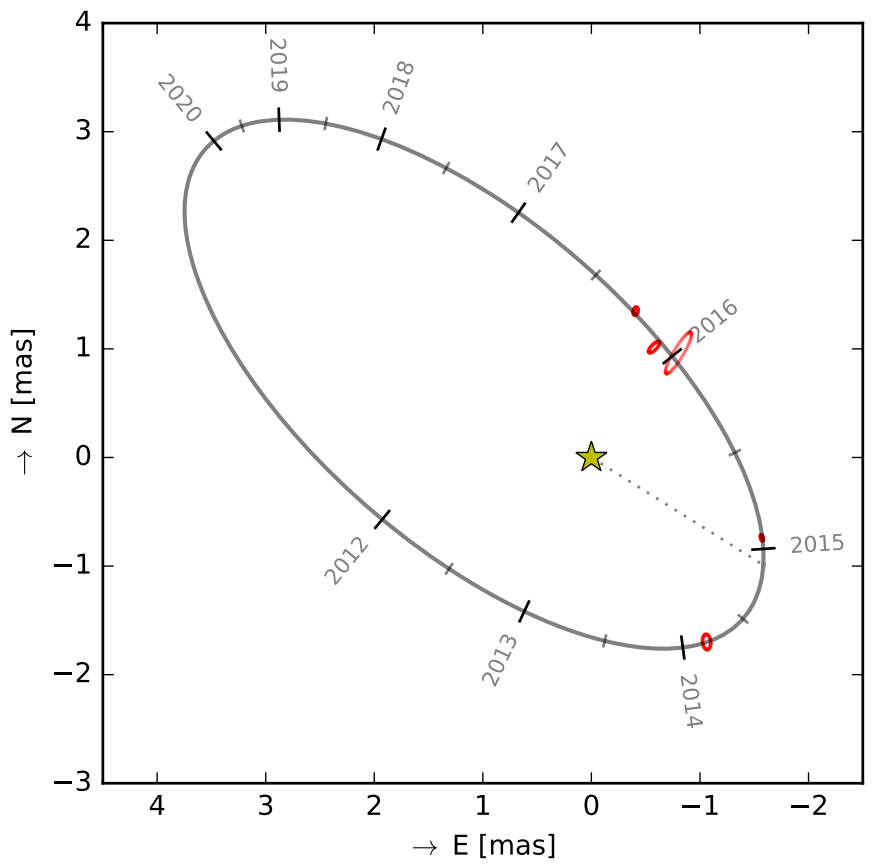

Fig. 4. Best orbit obtained to fit all our data points. The parameters of this orbit are given in Table 5 .

$5.4 \mathrm{kpc}$ to about $11 \mathrm{au}$. The longest periods thus correspond to the highest eccentricity, with the largest possible period ${ }^{6}$ having an improbable eccentricity close to 1 . For a small range of periods - roughly five to eight years - the eccentricity is quite moderate and below 0.2. These are not, however, the solutions which are the most likely in terms of $\chi^{2}$.

The other parameters are also not independent, and they vary in a very narrow range. Thus the time of periastron passage is constrained between 2013.2 and 2015.05, the later being valid for most long-period solutions. Only for the shortest periods did the periastron happen earlier. The inclination of the orbit on the plane of the sky is also quite narrowly defined, between 115 and $120 \mathrm{deg}$.

Assuming a distance of $5.4 \mathrm{kpc}$, we can also translate our constraints as a function of the total mass of the binary systems and see that our solutions imply a total mass range between 33.6 $M_{\odot}$ and $80 M_{\odot}$ (for the shortest orbits). The most favoured range (in terms of $\chi^{2}$ ) is the lowest one, i.e. roughly between 33.6 and $45 M_{\odot}$. Assuming a mass of $25 M_{\odot}$ for the primary LBV (see above), this would imply a companion mass between roughly 9 and $20 M_{\odot}$, i.e. a mass ratio between 0.36 and 0.8 .

In Fig. 5, we show the ellipses corresponding to $1 \sigma$ difference between the various orbits. If the size of these ellipses is much larger than the precision on our positions, then we should be able to distinguish between the different families. From the figure, it seems clear that by 2018 , we should already be able to much better constrain the orbit and tell if HR Car is a short- or long-period binary.

Depending on the orbital period and eccentricity, the possible velocity change that the primary would undergo over its orbit could be estimated. This of course depends crucially on the mass ratio in the system and, for illustration purposes, we have here assumed a value of 0.5 . As seen from the bottom panels of Fig. 6, the resulting semi-amplitude of the primary is in the

\footnotetext{
6 From an anthropocentric argument, the longest periods are also less favourable since they imply that we have been quite lucky to be observing the system now, at its closest approach.
}

range between a few to $22 \mathrm{~km} \mathrm{~s}^{-1}$ (for the shortest orbits). Given that HR Car is characterised by extremely variable lines over a $\mathrm{S}$ Dor cycle, as well as outflows of up to $150 \mathrm{~km} \mathrm{~s}^{-1}$, this orbital motion would be hard to detect, which would probably explain why HR Car has not yet been characterised as a spectroscopic binary.

Finally, it is important to realise that the binary companion we detected here has nothing to do with the possible B0 V companion inferred by White (2000) from radio data, since this companion is thought to be about two arcseconds away, i.e. a thousand times farther away than the one we detected.

\subsection{Size of the primary}

For the primary, we derive an angular $H$-band diameter of the primary of $0.38 \pm 0.08$ mas. At the distance of $5.4 \pm 0.4 \mathrm{kpc}$, this would translate in a radius of $220 \pm 60 R_{\odot}$, which is nominally too large for the radius of the $\mathrm{LBV}^{7}$. However, LBVs are generally surrounded by large envelope of ejected matter (e.g. Vamvatira-Nakou et al. 2015). In fact, HR Car is surrounded by a bipolar nebula whose lobes have a diameter of $0.65 \mathrm{pc}$, i.e. much larger than anything we measure here (Weis et al. 1997). We can thus assume that an optically thick wind around the photosphere of the star is being detected. Moreover, we see the star during its current S Dor minimum - its radius should be much larger during a maximum. We speculate that this is perhaps because we are in a minimum that we were able to detect such a faint companion - it was with a flux ratio of six when we discovered it and is now with a flux ratio of nine, but during a maximum, the flux ratio would be in the range of 30-40!

\subsection{Size and possible nature of the secondary}

We derive a rather constant angular diameter for the secondary companion of $0.85 \pm 0.20$ mas, translating to a radius of $500 \pm$ $150 R_{\odot}$, while at the same time, this object is about six to nine times fainter in the $H$-band than the primary LBV star. This seems a priori difficult to reconcile unless, perhaps, one considers the possibility that the secondary is a red supergiant. Using the current $V \sim 9$ mag of the combined HR Car system, and values of $V-H$ and bolometric corrections (Levesque et al. 2005; Davies et al. 2013) of red supergiants, it is easy to show that a typical red supergiant with an effective temperature of 3600$4000 \mathrm{~K}$ would have the right flux and size to fit the data. This type of star would have a mass below $15 M_{\odot}$ and would contribute to less than $1 / 10$ th of the total flux of the system in the $V$-band, explaining why it had escaped detection up to now. It remains to be seen if a binary system of a given age with a massive LBV and a less massive red supergiant can be reproduced by stellar evolutionary models, but this is beyond the scope of this paper.

Another possibility is that the secondary is out of equilibrium. The LBV was much larger around the year 2001 (around the maximum), so if the periastron happened close to this epoch $^{8}$, the secondary could have gone though the outer layers of the LBV, possibly leaving the secondary out of hydrostatic equilibrium.

7 Given the bolometric magnitude and temperature quoted in the Introduction, we derive a radius of $90 \pm 25 R_{\odot}$, much smaller than what is found here. Nevertheless, the radius we derive is about 2.5 larger, which is compatible with the size of the wind continuum emission region.

8 A possibility to consider if the orbital period is of the order of a few to $15 \mathrm{yr}$. 

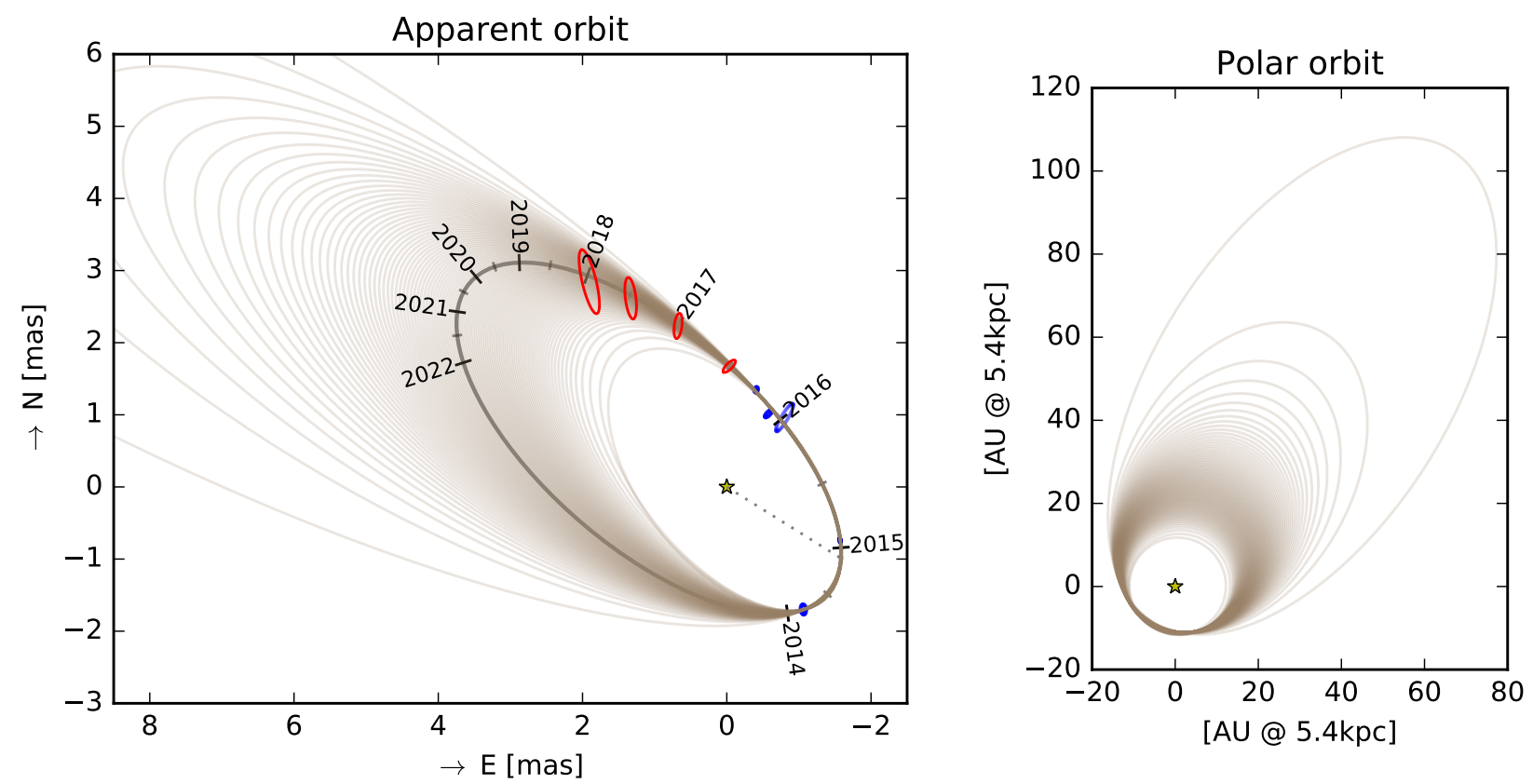

Fig. 5. Family of orbits that fit all our PIONIER data points. The left panel shows the observed points and the projected orbits, as well as $1 \sigma$ ellipses allowing to distinguish between the various orbits. A large number of orbits still fit the data, with the longer orbits being the more eccentric. The right panel shows the de-projected orbits, in absolute scale, assuming a distance of $5.4 \mathrm{kpc}$. All orbits from Fig. 6 are shown here.

Since we determine the sum of the radii of both components to be about 0.6 mas, this means that even at periastron, the separation between them is more than three times as much. The smallest Roche lobe radius for the secondary would be about 0.76 mas, which is much more than the radius we measure for the secondary. Hence, the secondary will never fill its Roche lobe.

We note, however, that the flux of the secondary varies with time - it appears to be decreasing from a relative contribution of $16 \%$ to $10 \%$ within the two-year window of our observations. This could mean that the primary is becoming brighter, but this is not confirmed by the AAVSO light curve which shows that in the $V$-band - the flux of HR Car only varied by $30 \%$ at most since January 2014. On the other hand, as shown earlier, the periastron passage took place most likely in late 2014 for a wide range of orbital periods - so it is possible that the flux of the secondary varies because of some interaction with its companion or because of some geometrical effect, and that it is now weakening as the companion moves further away from the primary. If this were the case, one should not try to relate the flux we measure to a bolometric magnitude of the secondary.

\subsection{Background flux}

Our best fit clearly requires the presence of some background flux, at the level of a few percents. With the current data we are unable to determine the size of the region that contributes to this background flux, but it has to be larger than the separation between the two components. It is tempting to relate this background flux to what AMBER detected, i.e. the $\mathrm{Br} \gamma$ emission region, although one should note that, in the continuum and at shorter wavelength, the wind extension must be much smaller than the line formation region.

Most interesting is the fact that the flux of the background has greatly increased since January 2016 - in fact, it almost tripled in about three months. It will be interesting to follow the evolution of this background flux and, in particular, try to understand how it relates to the orbital parameters.
We would like to stress, however, that it is possible that the background flux we measure is an instrumental effect, since there have been some claims that PIONIER possibly has a bias in the visibilities for bright objects performed with the HIGH gain. Until this can be fully disproved, we should beware of overinterpreting this background.

\subsection{Return to AMBER}

With the binary nature of HR Car confirmed and the system dimensions constrained with PIONIER, revisiting the original AMBER data offers further insights. The first PIONIER observation was simultaneous to the OHANA survey data and confirms that the phase signature in the $\mathrm{Br} \gamma$ emission marks an offset of the emission towards the secondary. Further, the OHANA observations show a clearly elongated wind, larger in the direction that is perpendicular to the line of sight between the components. Finally, the measured projected distance between the components in March 2014, 2 mas, is less than the extension of the Br $\gamma$ emission region.

It is quite straightforward to reconcile that description with the images evoked by SPH simulations of wind-wind interaction (if the secondary star has a wind), as for instance shown by Madura et al. (2013) for $\eta$ Car. The wind-wind interaction front may reduce the size of the emission region along the line of sight between the components and, at the same time, increases the local emissivity. As a prediction for an eventual detection of the orbital RV curve, it follows that, during the 2014 observations, the secondary was closer to Earth than the primary, so that the wind-wind interaction took place in the blue-shifted part of the primary's dense wind.

Although the orbit is not yet fully constrained, a few things are already clear. In the case of an eccentric system, with a rather long period of well over a decade, the 2014 observations must have been around periastron (within about a year) while the 

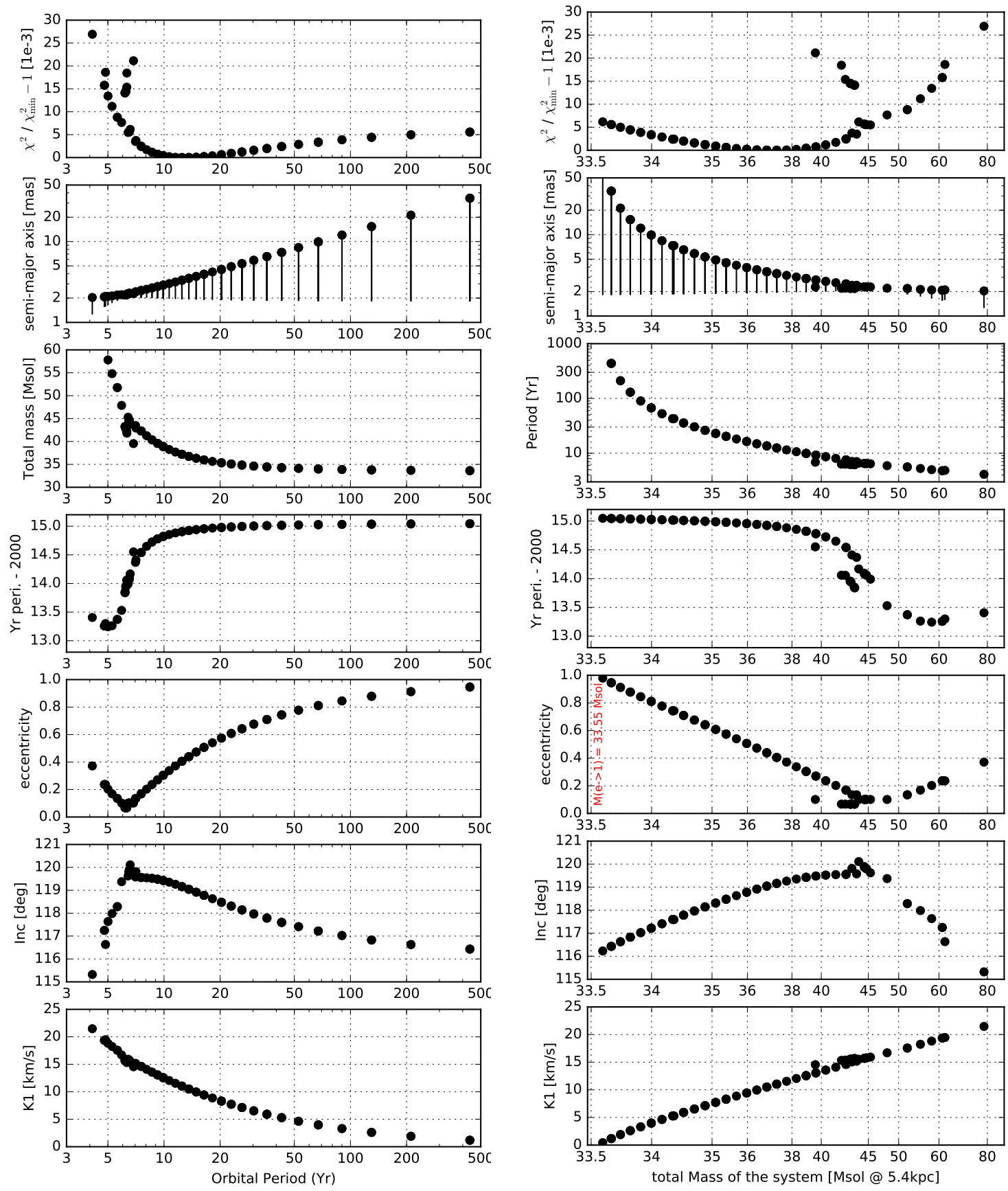

Fig. 6. Results from our solution grid-search to fit all the PIONIER data. The left panels show the parameters plotted as a function of the orbital period, while the right ones show them as a function of the total mass of the binary, assuming a distance of $5.4 \mathrm{kpc}$. The upper panels show the difference in ratio of the reduced $\chi^{2}$; the second the semi-major axis, with the line indicating the periastron distance; third is either the mass as a function of period or vice-versa; fourth panels are the time of periastron passage (-2000); fifth, the eccentricity; the sixth show the inclination of the orbit, while the bottom panels show the radial velocities of the primary assuming a mass ratio of 2 . We note the degeneracy in orbital solutions near zero eccentricity. These orbits have slightly worse $\chi^{2}$ than the others at same semi-major axis.

2010 observations were not. The projected periastron distance between both components is then the distance measured, about 2 mas. This would be one possibility for explaining the absence of a phase signature and the more extended NE-SW wind in 2010, namely that in 2010 the undisturbed configuration of the wind was observed, which is similar to the one measured in 2014 along the NW-SE axis.

In the case of a circular orbit, which would have a shorter period of up to about a decade, the time difference between the observations is such that the wind-wind interaction front might just have rotated by about one quarter, so that the $2010 u, v$ position of panel e is equivalent to the $2014 u, v$ position $^{9}$ of panel $\mathrm{d}$.

\section{Conclusions}

We have obtained interferometric observations of the LBV HR Car that clearly reveals its binary nature and detected the orbital motion over a period of two years. It is still not possible

9 Or its conjugated point, as since there is no phase signature, this is degenerate. 
to derive the orbital period that could be of the order of a few to several tens of years and the separation of the order of 10$270 \mathrm{au}$, but with the constraint that the largest orbit must also be the most eccentric, with a periastron distance most likely fixed around 2 mas or $11 \mathrm{au}$. If the eccentricity is small and the orbit turns out to be of the order of five to ten years, HR Car would be the second binary LBV presenting all the hallmarks and properties that make $\eta$ Car truly such a unique object, but with components of much smaller masses. We note, however, that no giant eruption has ever been recorded for HR Car, unlike the one of the 1840s of $\eta$ Car, and that estimates of the ejecta mass surrounding HR Car are more of the order of $1 M_{\odot}$ (White 2000), much smaller than that seen around $\eta$ Car.

Apart from highlighting the possible role of binarity in the formation and/or evolutions of LBVs, the fact that HR Car is a binary is essential since it allows us to derive the masses of stars, which is very useful for comparing stellar evolutionary models. For now, we constrain the most likely range of total masses as being 33.5-45 $M_{\odot}$.

AMBER has shown that the emission line-forming region of $\mathrm{Br} \gamma$ is larger than the minimum projected separation of the components of 2 mas, measured by PIONIER. Hence HR Car must undergo wind-wind interaction detectable in $\mathrm{Br} \gamma$, and probably in $\mathrm{H} \alpha$ as well. Whether the interaction is permanent, in case of a circular orbit, or phase-dependent at periastron, in the case of an eccentric orbit, cannot be decided with the current AMBER data, although the increase in background flux seen in the PIONIER data seem to favour the latter (if it proves to be non-instrumental). In either case, however, the HR Car system is considerably simpler than its much better known, nearby LBVsibling, $\eta$ Car, and probably much easier to constrain, model, and ultimately understand. In particular, in the case of an eccentric orbit the next periastron would offer an excellent opportunity for a concerted multi-wavelength, multi-technique campaign to provide constraints for theoretical modelling.

Whatever interaction happens in the system of HR Car now, in the minimum phase of its S Dor cycle, it must be very different when it is at maximum. In the maximum of an S Dor cycle, the primary, which is well separated from the secondary now (even at the closest distance) will possibly become close or exceed its Roche-lobe radius and maybe even become large enough for the secondary to pass through the primary's outer layers. Recently, two S Dor cycles have been observed for HR Car, with maxima around 1991 and 1999 (see, e.g., Szeifert et al. 2003) or 2001 (as indicated by the AAVSO data). Once the orbit is better constrained, it will be possible to see how these dates relate to the orbital parameters; whether one should have expected strong interaction between the components and, in particular, what to expect in the next $\mathrm{S}$ Dor maximum phase.

We will continue to monitor HR Car with the PIONIER instrument to try to settle as soon as nature allows us the orbital period of this interesting binary. From the current modelling, in 2018, we should already be able to distinguish between the main families of solutions. This combined with a precise Gaia distance should also constrain the total mass of the system. We encourage spectroscopic monitoring of this target to try to derive the associated spectroscopic orbit, although we understand that this won't be a task for the faint-hearted.

Acknowledgements. It is a pleasure to thank Steve Ertel for taking the data in January 2015 in delegated Visitor Mode. The archival AMBER observation was obtained under ESO Prog. ID 085.D-0490.

\section{References}

Berger, J., Zins, G., Lazareff, B., et al. 2010, in Proc. SPIE, 7734, 99 Chelli, A., Utrera, O. H., \& Duvert, G. 2009, A\&A, 502, 705

Chini, R., Hoffmeister, V. H., Nasseri, A., et al. 2012, MNRAS, 424, 1925

Conti, P. S. 1984, Observational Tests of the Stellar Evolution Theory, 105, 233 Davies, B., Kudritzki, R.-P., Plez, B., et al. 2013, ApJ, 767, 3

Gallagher, J. S. 1989, IAU Colloq. 113: Physics of Luminous Blue Variables, 157,185

Gallenne, A., Mérand, A., Kervella, P., et al. 2015, A\&A, 579, A68

Groh, J. H., Damineli, A., Hillier, D. J., et al. 2009, ApJ, 705, L25

Hohle, M. M., Neuhäuser, R., \& Schutz, B. F. 2010, Astron. Nachr., 331, 349

Humphreys, R. M., \& Davidson, K. 1994, PASP, 106, 1025

Humphreys, R. M., Weis, K., Davidson, K., \& Gordon, M. S. 2016, ApJ, 825, 64 Hutsemékers, D., \& van Drom, E. 1991, A\&A, 248, 141

Le Bouquin, J.-B., Berger, J.-P., Lazareff, B., et al. 2011, A\&A, 535, A67

Levesque, E. M., Massey, P., Olsen, K. A. G., et al. 2005, ApJ, 628, 973

Lobel, A., Groh, J. H., Martayan, C., et al. 2013, A\&A, 559, A16

Machado, M. A. D., de Araújo, F. X., Pereira, C. B., \& Fernandes, M. B. 2002, A\&A, 387, 151

Madura, T. I., Gull, T. R., Okazaki, A. T., et al. 2013, MNRAS, 436, 3820

Martayan, C., Lobel, A., Baade, D., et al. 2012, in Circumstellar Dynamics at High Resolution, ASP Conf., 464, 293

Martayan, C., Lobel, A., Baade, D., et al. 2016, A\&A, 587, A115

Petrov, R. G., Malbet, F., Weigelt, G., et al. 2007, A\&A, 464, 1

Rivinius, T., Boffin, H. M. J., de Wit, W. J., et al. 2015, IAU Symp., 307, 295

Rivinius, T., de Wit, W. J., Demers, Z., Quirrenbach, A., \& the VLTI Science Operations Team 2016, ArXiv e-print [arXiv: 1602 .03457]

Sana, H., de Mink, S. E., de Koter, A., et al. 2012, Science, 337, 444

Sana, H., Le Bouquin, J. -B., Lacour, S., et al. 2014, ApJS, 215, 15

Smith, N. 2011, MNRAS, 415, 2020

Smith, N., \& Tombleson, R. 2015, MNRAS, 447, 598

Smith, N., Gehrz, R. D., Hinz, P. M., et al. 2003, AJ, 125, 1458

Szeifert, T. and Kaufer, A., Crowther, P. A., Stahl, O., \& Sterken, C. 2003, IAU Symp., 212, 243

Tallon-Bosc, I., Tallon, M., Thiébaut, E., et al., 2008, SPIE 7013, 701323

Tetzlaff, N., Neuhäuser, R., \& Hohle, M. M. 2011, MNRAS, 410, 190

Vamvatira-Nakou, C., Hutsemékers, D., Royer, P., et al. 2015, A\&A, 578, A108

van Genderen, A. M., The, P. S., Heemskerk, M., et al. 1990, A\&AS, 82, 189

van Genderen, A. M., Robijn, F. H. A., van Esch, B. P. M., \& Lamers, H. J. G. 1991, A\&A, 246, 407

van Leeuwen, F. 2007, A\&A, 474, 653

Vink, J. S. 2012, Astrophys. Space Sci. Libr., 384, 221

Weis, K., Duschl, W. J., Bomans, D. J., Chu, Y.-H., \& Joner, M. D. 1997, A\&A, 320,568

White, S. M. 2000, ApJ, 539, 851 